\title{
Optimum Integral Design for Maximizing the Field in Short Magnets
}

\author{
Ramesh Gupta
}

\begin{abstract}
An Optimum Integral Design is introduced for $\operatorname{cosine}(n \theta)$ coils where the entire end-to-end length of the coil generates field with the dilution from ends practically eliminated. The benefits of such a design are particularly significant in short magnets where the overall coil length is comparable to or a few times the coil diameter. The integral field strength is further enhanced since the design allows a larger number of turns than in typical magnet coils. In this concept, the ends and body harmonics are optimized together to create an integral $\operatorname{cosine}(n \theta)$ azimuthal current distribution. The concept was initially developed for wire/cable wound magnets where the bend radius of turns in the ends can be small. However, the benefit of this general approach can be applied to cable magnets as well. The magnetic design of a corrector dipole for the AGS helical magnet, which was recently built and tested, is presented as one of several examples. The other examples include a few sub-compact designs: a dipole with coil length less than a coil diameter, a quadrupole with coil length less than a coil radius, etc. Apart from generating a large integral field for the given length, the computed integral field harmonics in these designs are only a few parts in 10,000 at $2 / 3$, of the coil radius.
\end{abstract}

Index Terms-Corrector, magnet designs, medical applications, short magnets.

\section{INTRODUCTION}

$\mathrm{T}$ HERE are an increasing number of applications in accelerators and in the medical industry where the available space for magnets is rather limited. In short-length conductor dominated magnets, where the mechanical length of the coil is comparable to or a few times the coil diameter (aperture), the ends determine the magnetic design and the length of the magnet itself. In conventional conductordominated dipole magnets, the mechanical length of each end is 1.5 to 2 times the coil diameter (see Fig. 1) [1]. The average field over the length of these ends is generally about $1 / 2$ to $2 / 3$ of that in the magnet cross-section (straight section). This means that such designs are not well suited for very short dipoles since the two ends themselves will use a length that is about three times the coil diameter. Moreover, the integral field in these end regions is about $2 / 3$ (or even less in many cases) of that in the same length of straight section.

Manuscript received October 3, 2004.

Ramesh Gupta is associated with Brookhaven National Laboratory, Upton, NY 11973 USA. E-mail address of the corresponding author: gupta@bnl.gov.

This work is supported by U.S. Dept. of Encrgy under contract No. DEAC02-98CH10886.
The increase in length comes primarily from the presence of end spacers (see Fig. 1) that must be used to minimize the field harmonics and also to reduce the peak field on the conductor in superconducting magnets. The average field in the end section is smaller than the field in the same length of the straight section. In very long magnets the computed field in most of the straight section is constant and is referred to as two-dimensional (2D) field, as it can be computed using 2D formulations. In short magnets, the field in the middle of short straight section may be different (generally smaller) than this 2D field. The effective magnetic length, defined here as the field integrated over the length of the magnet divided by the 2D field, is smaller than the coil length. The typical loss in the effective magnetic length over the coil length due to ends is of the order of a coil diameter in dipoles, a coil radius in quadrupoles, etc.

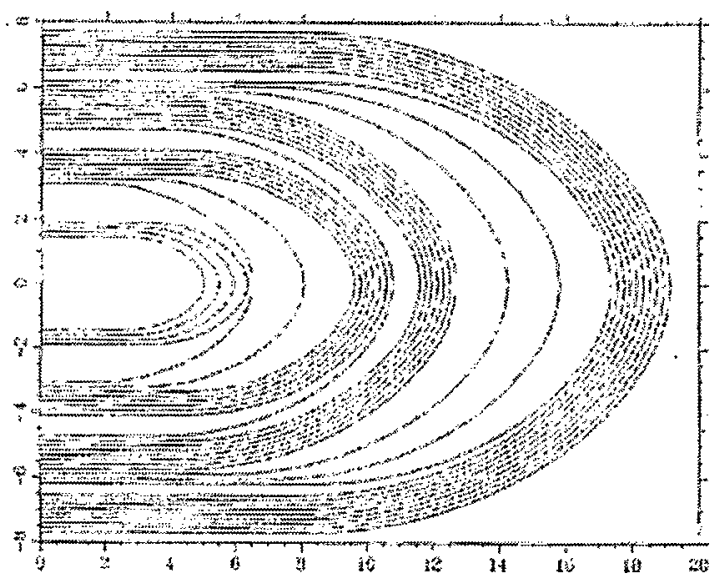

Fig.1. Layout of the outer edge of the end turns in $8 \mathrm{~cm}$ aperture RHIC dipole. The horizontal axis is axial position (in $\mathrm{cm}$ ) and the vertical axis is the half circumference (in $\mathrm{cm}$ ) of the outer edge of the upper coil. The width of the cable is about onc $\mathrm{cm}$.

The physical space taken by the turns in the end itself is of the order of a diameter in typical dipoles and of the order of a radius in typical quadrupoles. The exception to this is the "bedstead ends", which, however, are not desirable in superconducting magnets and are associated with a large buildup in radial direction [2]. Thus, in very short dipoles (with coil length similar to coil diameter), one would have to significantly reduce the number of turns in the cross-section to reduce the relatively large physical space taken by the turns in the end. This, however, also brings a large reduction in the 2D and in the integral field. 
B. Parker has proposed a clever Serpentine coil design [3] that eliminates the need for optimizing the magnet ends for field harmonics. The Serpentine design offers several other advantages as well. An example of this design is shown in Fig. 2. Here a coil of any number of poles is continuously wound with the end-turns for each layer located only on one end of the pole (in two layers, this end is alternated to essentially eliminate any solenoid field). Since each turn is successively moved axially by a similar ( wire diameter) length, the length of every turn remains the same. In the limiting case where the bend radius of each turn in the end approaches zero, the integral field and the field harmonics in the entire coil will be the same as those in $2 \mathrm{D}$ section, even when no end-spacers are used. Therefore, to a good approximation, the integral field will be given by the " $2 \mathrm{D}$ field" multiplied by the "coil length minus the space taken by the end turns". Therefore, the loss in effective magnetic length is still about a coil diameter for dipoles and a coil radius for quadrupoles.

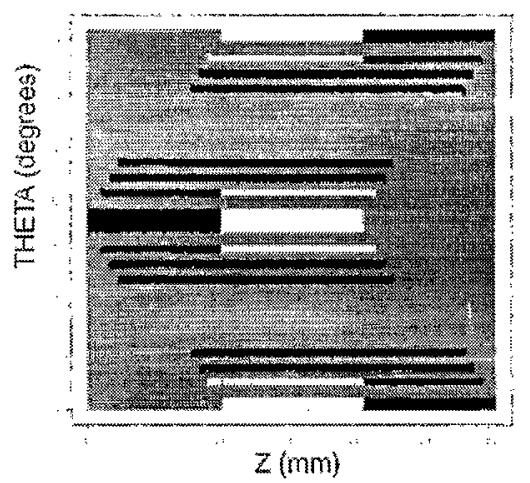

Fig.2. An example of 2-laycr "Serpentine Coil Design" [3].

The loss in effective magnetic length may be attributed to the fact that in both $\operatorname{cosine}(n \theta)$ and Serpentine designs the midplane turns that contribute most to the field do not span the entire coil length.

End design (and also cross-section design) based on stream function has been proposed by P. Walstrom where a few midplane turns (turns that are near the midplane) are extended to the end of the coil [4]. However, the overall fill factor (fraction of area filled with conductor) is similar to that in conventional designs and so is the integral transfer function.

\section{Optimum InTEgral DESign}

An Optimum Integral Design is introduced here where the midplane turns essentially span the entire length of the coil. This and the design principles outlined below eliminate the loss in integral field due to ends. The design was initially developed for magnets made with conductor that can have a bend radius much smaller than the coil radius (for example, the magnets made with small diameter wire). The general concept, however, can be extended to cable magnets with larger bend radii. The actual magnetic designs presented in the subsequent sections use bend radii in the ends of magnets that have been already built and successfully tested. The Optimum Integral Design is explained in the following paragraphs.
Conventionally, in designing most conductor dominated (2n) multi-pole magnets, first the coil cross section is optimized and then the ends for minimizing field harmonics and peak field on the conductor. This 2-step optimization creates a magnet with low integral harmonics but, unfortunately, also one that has a magnetic length that is smaller than the coil length, typically by a coil diameter/(n).

In the proposed Optimum Integral Design, the length of the midplane turn is the same as the coil mechanical length (endto-end) with bend radius of turns in the ends approaching zero. If there are no spacers in the ends or in the straight section, and if all turns are spaced equally, then the length of successive turns decreases linearly in going from midplane to pole. One way to obtain an ideal current distribution (in integral sense) is to modulate the length of each turn so that it is proportion to cosine $(n \theta)$. In a more practical approach, the integral modulation is obtained with the help of a computer program after distributing a total of " $N$ " turns in a few end blocks and/or in a few cross-section blocks. The size of spacers between the blocks is optimized to achieve an integral distribution varying azimuthally as:

$$
I(\theta) L(\theta)=I_{o} \sum_{i}^{N} L_{i}(\theta) \propto I_{o} L_{o} \cdot \cos (n \theta)
$$

Since the cosine theta modulation is normalized to the current $I_{o}$ times the length $L_{o}$ (end-to-end coil length), this equation suggests that the integral field of the magnet may be closer to typical 2D field times the mechanical length of the coil $\left(L_{o}\right)$. This is a significant improvement over the designs discussed in the previous section where the loss in effective magnetic length from $L_{o}$ was about a coil diameter $/(n)$.

\section{MAGNET DESIGNS BASED ON OPTIMUM INTEGRAL DESIGN}

In short magnets, where the coil length is only a few times the coil diameter, an integral description and optimization of the field may be more appropriate. It is possible to optimize a cosine $(n \theta)$ type integral distribution with spacers (wedges) only in the straight section (in all but second example in this section), or with spacers only in the ends (see second example). However, in general, one would take advantage of both end and straight section spacers, as needed.

Several examples are presented below. The first example is for the AGS helical corrector dipole, which has been optimized, built and tested. Other coil designs are given here for illustration purpose only and are not fully optimized. They are meant to show that the concept can generate compact and efficient designs.

\section{A. AGS Helical Corrector Dipole}

The corrector package for the superconducting AGS helical dipole $[4,5]$ requires a solenoid and two corrector dipole magnets. The coils are wound on the bore tube inside the helical dipole to minimize overall length. The space restriction further required the coils to have small length. This led to the development of the Optimum Integral Design.

The required integral field strength of 0.008 Tesla.meter is obtained in a single layer coil having an inner diameter of 
$182.8 \mathrm{~mm}$ and end-to-end length of $300 \mathrm{~mm}$. The diameter of the NbTi superconducting wire is $0.33 \mathrm{~mm}$ and the turn-toturn spacing is $0.635 \mathrm{~mm}$ to allow adequate insulation. The bend radius of turns in the end is $6.35 \mathrm{~mm}$. The yoke inner diameter is $300.4 \mathrm{~mm}$. The design gave a magnet with a $-180 \%$ quench margin with the $3 \mathrm{~T}$ helical dipole field superimposed on it. Since the applied $3 \mathrm{~T}$ field is over an order of magnitude more than the field created by the corrector dipole, the peak field of the corrector coil itself does not play a major role in determining the quench margin. The design integral field is obtained at $25 \mathrm{~A}$, which gives the $50 \mathrm{~A}$ HTS current leads a sufficient margin as well.

An OPERA-3d model [2] of the optimized design is shown in Fig. 3 and the coil as built in Fig. 4. One can see that the turns at the midplane use almost the entire end-to-end coil length. The design uses 199 turns per quadrant with the turns occupying $88 \%$ of the circumference. This is much more than the $65 \%$ in a typical cross-section. Moreover, there is only one small spacer (3.09 degrees after 24 turns) in the cross section and none in the ends. The pole angle is 81.8 degrees; any coil turn after that angle would contribute little to the dipole field. The space at the pole is about the minimum required. This coil has a much larger area filled with the conductor than that in conventional designs and is about the maximum one can fill. Thus this is about the most integral field strength one can obtain with or without good field harmonics.

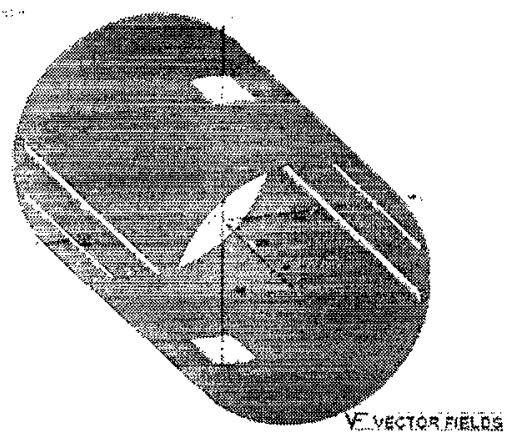

Fig. 3. OPERA-3d model of the AGS corrector dipole based on the Optimum Integral Design.

The computed integral field harmonics at $60 \mathrm{~mm}$ radius $(\sim 2 / 3$ of the $90.8 \mathrm{~mm}$ coil radius) for this optimized design are given in Table I. These harmonics are much better than typically required in corrector dipoles and easily satisfied the design specifications. Two such corrector magnets have been built and tested for quench performance and field harmonics. The quench current test was limited by the power supply ( 45 A) and was well above ( $80 \%)$ the design current of $25 \mathrm{~A}$. The measured harmonics agreed with the computed harmonics within measurement errors.

The harmonics were optimized with the computer code "MINXEND [7]" that was exclusively written to minimize harmonics in Optimum Integral Design. It also creates an OPERA-3d input file that was used to generate various OPERA-3d models shown in this paper. Another important tool in building this magnet was the program "flatpattergen [8]" that creates a wiring file to drive a computer driven multiaxis machine [9] that lays wires directly on the bore tube as shown in Fig. 4. The program flatpatterngen also computes and minimizes the allowed and non-allowed harmonics for the complete wire layout (including the leads).

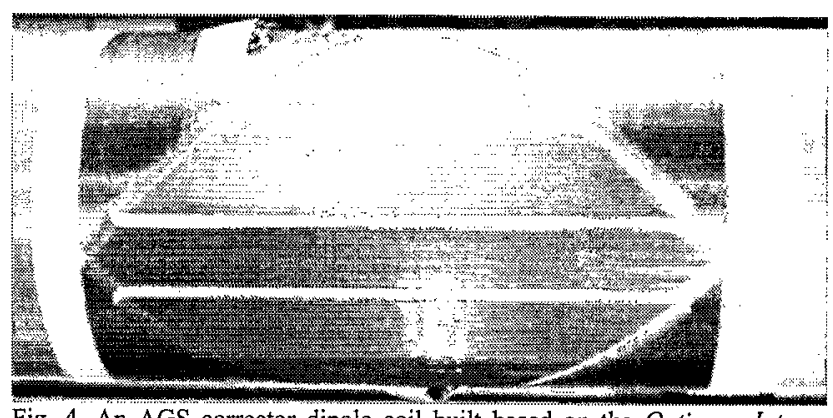

Fig. 4. An AGS corrector dipole coil built based on the Optimum Integral Design. One can note that the midplane turns span almost the full end-to-cnd coil length and the coil has a high fill factor of supcrconducting wircs.

TABLE I

COMPUTED INTEGRAL FIELD HARMONICS IN THE AGS CORRECTOR DIPOLE DESIGN AT A REFERENCE RADIUS OF $60 \mathrm{MM}$. THE COIL RADIUS IS $90.8 \mathrm{MM}$. NOTE $b_{2}$ IS SEXTUPOLE MUTLIPLIED BY $10^{4}$ (US CONVENTIONS).

\begin{tabular}{lcccccc}
\hline Integral Field (T.m) & $b_{2}$ & $b_{4}$ & $b_{6}$ & $b_{s}$ & $b_{10}$ & $b_{12}$ \\
\hline $0.0082(a) 25 \mathrm{~A}$ & 0.4 & 0.8 & -4.7 & 4.1 & 5.3 & 2.4 \\
\hline
\end{tabular}

\section{B. A Dipole Design Optimized with End Spacers Only}

In this section, we present a dipole design that has been optimized with end spacers only, i.e. the cross-section does not have any spacers (or wedges). The design is based on a two-layer coil using the same wire that has been used in the AGS corrector dipole described in the previous section. The dipole has an aperture of $\sim 224 \mathrm{~mm}$ and a length of $500 \mathrm{~mm}$ (about twice the average coil diameter). The harmonic optimization goal is to obtain integral harmonics of less than five units at $50 \mathrm{~mm}$ radius. The design has 250 turns in the inner layer (with end turn configuration of 200, 30 and 20) and 220 turns in the outer layer. There is no spacer in the outer layer. The pole angle is 80.9 degree for the inner layer and 70.7 degree for the outer layer. The configuration is chosen for its lower peak field. An OPERA-3d model of the design is shown in Fig. 5 and the computed harmonics in Table II.

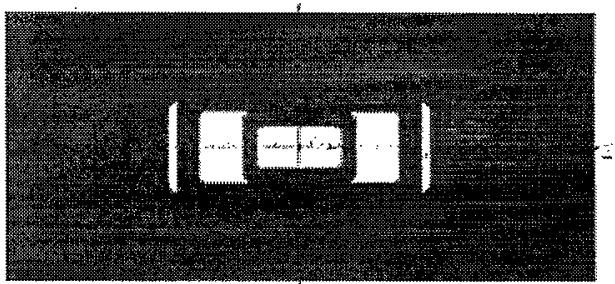

Fig. 5. OPERA-3d model of a 2-layer coil (seen from the top/pole) based on the Optimum Integral Design. It has no spacers (wedges) in the cross-scetion and has only two each in the either end of the inner layer.

TABLE II

COMPUTED INTEGRAL HARMONICS IN A DIPOLE THAT IS OPTIMIZED WITH

TwO END SPACERS ONLY AS NO STRAIGHT SECTION SPACER WAS USED.

THE REFERENCE RADIUS IS $50 \mathrm{MM}$ AND THE COIL RADIUS IS $111.9 \mathrm{MM}$. NOTE $b_{2}$ IS SEXTUPOLE MUTLIPLIED BY $10^{4}$ (US CONVENTIONS).

\begin{tabular}{lcccccc}
\hline Integral Field (T.m) & $b_{2}$ & $b_{4}$ & $b_{k}$ & $b_{n}$ & $b_{10}$ & $b_{12}$ \\
\hline $0.247(a) 27 \mathrm{~A}$ & 3.0 & 4.0 & 4.5 & -0.6 & 0.1 & 0.0 \\
\hline
\end{tabular}


Comparison of the two optimized coil configurations (a) based on Optimum Integral Design (Fig. 5) and (b) based on Serpentine design (Fig. 2) reveals the following. In a coil that is smaller in length by $20 \%$ (500 $\mathrm{mm}$ instead of $610 \mathrm{~mm}$ ), the Optimum Integral Design generates over 10\% more integral field $(0.247$ T.m instead of 0.224 T.m) than the Serpentine design at the same current.

\section{A Dipole with Coil Length Less Than Coil Diameter}

In this section, we investigate the possibility of making very short dipoles based on the Optimum Integral Design. We choose a coil diameter of $200 \mathrm{~mm}$ and a coil length of $\sim 175$ $\mathrm{mm}$. When the coil length is less than half the circumference of a dipole coil, the coil length limits the fill factor in the cross-section. In addition, a space must be left at the pole for a splice. This gives a maximum fill factor of $\sim 61 \%$. We used empty space in the cross-section to demonstrate that six small spacers (wedges) can make the first six allowed harmonics nearly zero (see Table III). The gap at the pole is made larger in the angular direction and smaller in the axial direction to obtain a high integral transfer function while allowing sufficient space for the splice. The wire and most other parameters in this design are the same as those outlined in the first example (AGS corrector dipole). An OPERA-3d model is shown in Fig. 6. A large integral transfer function is obtained since the midplane turns span the entire end-to-end coil length.

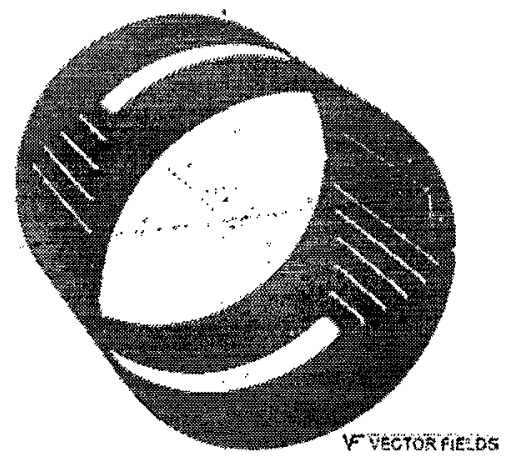

Fig. 6. OPERA-3d model of a short length dipole based on the Optimum Integral Design. Coil length is $\sim 175 \mathrm{~mm}$ and coil diameter is $200 \mathrm{~mm}$.

\section{TABLE III}

COMPUTED INTEGRAL FIELD HARMONICS FOR A SHORT DIPOLE (COIL LENGTH < DIAMETER) AT A RADIUS OF $66.6 \mathrm{MM}$. THE COIL RADIUS IS 100 MM. NOTE $b_{2}$ IS SEXTUPOLE MUTLIPLIED BY $10^{4}$ (US CONVENTIONS).

\begin{tabular}{lcccccc}
\hline \hline Intcgral Field (T.m) & $b_{2}$ & $b_{4}$ & $b_{6}$ & $b_{s}$ & $b_{t 1}$ & $b_{l_{2}}$ \\
\hline $0.00273(025 \mathrm{~A}$ & 0.0 & 0.0 & 0.0 & 0.0 & 0.0 & 0.0 \\
\hline
\end{tabular}

\section{A Quadrupole with Coil Length Less Than Coil Radius}

In this section, we carry out a similar exercise for a quadrupole. The coil diameter is $200 \mathrm{~mm}$ and coil length is 90 mm (less than half the radius). In a quadrupole, the coil length limits the fill factor in the cross-section when it becomes less than one-fourth of the circumference. We used six spacers (wedges) in the cross-section to make the first six allowed harmonics nearly zero. Once again, a large integral transfer function is obtained since the midplane turns span the entire end-to-end coil length.

\section{E. A Sextuupole with Coil Length 1/3 of Coil Diameter}

We carried out a similar exercise for a $200 \mathrm{~mm}$ aperture sextupole having an end-to-end coil length of $66 \mathrm{~mm}$. This is $\sim 1 / 3$ of diameter. We were again able to get a design with low harmonics and a good integral transfer function.

\section{F. Approach in Long Magnets Built with Rutherford Cable}

In long accelerator magnets (length an order of magnitude more than the coil diameter), it is desirable to decouple the optimization of ends and cross-section. Therefore, the Optimum Magnet Design approach cannot be applied in a manner used in previous sections. However, the general approach and philosophy of Optimum Integral Design will reduce the loss in integral field due to ends. In the conventional ends made with the "Rutherford Cable", the bend radius is smallest for the pole turns and largest for the midplane turns (see Fig. 1). The length of the turns near the midplane is less than the end-to-end coil length. In an approach based on the Optimum Integral Design, the turns near the midplane will have as small a bend radius as the turns near the pole and the midplane turns will be longer.

\section{CONCLUSION}

The proposed Optimum Integral Design concept offers a solution that allows magnets with large integral field and low integral harmonics to be built in a short length. This practically eliminates the loss of integral field due to ends. The design opens a new window for building very short magnets. Proof of principle examples of a dipole with length shorter than coil diameter, a quadrupole with length shorter than coil radius and sextupole with length shorter than one third of coil diameter have been presented.

\section{ACKNOWLEDGMENT}

We appreciate discussions with J. Escallier, A. Jain, B. Parker, P. Thompson, P. Wanderer and E. Willen. Magnetic measurement data of corrector dipole for AGS helical magnet were provided by A. Jain.

\section{REFERENCES}

[1] S. A. Kalın, ct al., "Calculations of Magnetic Ficld for the End Design of the RHIC Arc Dipole," in Proc. of 1993 Particle Accelcrator Conference, pp 2754-2756.

[2] Vector Ficlds, Inc., http://www.vectorficlds.com/.

[3] B. Parker, BNL, Private Communication (2003).

[4] P. Walstrom, "Design of End Turns in Current-dominated Dipole and Quadrupole Magnets for Ficlds with Low Higher Harmonic Content," in Proc. of Europcan Particle Accclerator Conference (2002).

[5] T. Roser, ct al., "Accelcration of Polarized Proton Beams Using Multiplc Strong Partial Sibcrian Snakes", in Proc. of European Particlc Accelerator Conference (2004).

[6] R. Gupta, et al., "Magnetic Design of A Superconducting AGS Snake," in Proc. of Particle Accelcrator Conference (2003).

[7] R. Gupta, "Optimum Integral Design for Maximizing Ficld in Short Magncts, " Magnet Division Note No. 634-37 (2003). Unpublished.

[8] P. Thompson, BNL. Private Communication (2003).

[9] B. Parker, ct al., "HERA Luminosity upgrade supcrconducting magnct production at BNL, in Proc. of ASC2000, pp 1518-1521. 


\section{DISCLAIMER}

This report was prepared as an account of work sponsored by an agency of the United States Government. Neither the United States Government nor any agency thereof, nor any of their employees, nor any of their contractors, subcontractors or their employees, makes any warranty, express or implied, or assumes any legal liability or responsibility for the accuracy, completeness, or any third party's use or the results of such use of any information, apparatus, product, or process disclosed, or represents that its use would not infringe privately owned rights. Reference herein to any specific commercial product, process, or service by trade name, trademark, manufacturer, or otherwise, does not necessarily constitute or imply its endorsement, recommendation, or favoring by the United States Government or any agency thereof or its contractors or subcontractors. The views and opinions of authors expressed herein do not necessarily state or reflect those of the United States Government or any agency thereof. 